\title{
A Stochastic Optimization Model for Real-Time Operation of Reservoirs Using Uncertain Forecasts
}

\author{
Bithin Datta ${ }^{1}$ \\ Department of Civil Engineering, University of Washington \\ MARK H. HoucK \\ School of Civil Engineering, Purdue University
}

\begin{abstract}
A real-time operation model primarily useful for daily operation of reservoirs is developed. This model is based on a chance constraint formulation and assumes a particular form of the linear decision rule. It uses the conditional distribution function (CDF) of actual streamflows conditioned on the forecasted values. These CDF's are constructed by incorporating the statistical properties of forecast errors for different time steps. The objective considered is the minimization of weighted probable deviations from storage and release targets. These weights are surrogates for the actual loss functions, and the probable deviations are functions of the reliability levels specified in the model. With the use of target values for release and storage, this model is capable of using a release policy that is a subset of a seasonal policy and overcomes the myopic (short-sighted) nature of operation. Simulation of actual operation, using this model for a hypothetical reservoir, demonstrates the feasibility and efficiency of this approach. This model is shown to be applicable for a system of reservoirs. The restrictions associated with the use of a linear decision rule are shown to be invalid for this model.
\end{abstract}

\section{INTRODUCTION}

Fundamental distinctions exist between optimization models intended for planning and those intended for shortterm or real-time operation of reservoirs. These distinctions are based on the type of information provided to these models and the goals and objectives to be satisfied. Long-term planning models should incorporate seasonal hydrologic data and long-term benefit functions. Long-term planning targets are obtained as outputs from these models.

Fór real-time operation, where decisions are made relatively quickly and are based on short-term information, decisions regarding release should be dependent on the starting reservoir storage, penalities for deviations from planned targets, and short-term forecasts. When dealing with very small time steps such as 1 hour, the hydrologic forecasts have very little uncertainty, and it should be possible to use these forecasts as deterministic inputs to an optimization model. This paper does not address the problem of minute-by-minute operation, however. The model described here emphasizes the incorporation of uncertainties inherent in short-term (for example, 12hour, 1-day, 3-day etc.) hydrologic forecasts into the decisionmaking process. Reliability measures of the system performance are developed on the basis of the forecasts' errors or hydrologic uncertainties.

\section{Background}

Some of the past research dealing with real-time operation of reservoirs is reported in Jamil Windsor [1973], Trott and Yeh [1973], Chu and Yeh [1978], Yeh et al. [1979], Toebes and Rukvichai [1978], Fults and Hancock [1972], Becker and Yeh [1974], Becker et al. [1976],

\footnotetext{
${ }^{1}$ Presently at Water Resources Management Laboratory, Engineering Experiment Station, University of Arkansas.

Copyright 1984 by the American Geophysical Union.

Paper number 4 W0694.

0043-1397/84/004W-0694\$05.00
}

Tennessee Valley Authority [1974], Houck [1981], Shane and Gilbert [1980], Yazicigil et al. [1983], and Sigvaldason [1976].

Useful discussion of the approaches reported in some of these papers can be found in Yeh [1982] and in Toebes and Rukvichai [1978]. Sigvaldason [1976] proposed a simulation model for the real-time operation of a multireservoir system by using a penalty function approach. Yazicigil et al. [1983] extended this simulation approach and presented a unified screening and simulation approach for the Green River Basin reservoir system. Houck [1981] commented on the sensitivity of the penalty functions used for real-time operation and proposed a suitable form of the objective function to achieve an operation policy that conformed more closely to a hypothetical, ideal operation policy. Shane and Gilbert [1980] proposed the combined use of simulation and optimization methods for a weekly time-step reservoir system scheduling model for the Tennessee Valley reservoir system.

No specific review of literature related to the application of chance-constrained programming methods to reservoir systems planning or operation is made here. Interested readers may refer to Hogan et al. [1981], ReVelle et al. [1969], Joeres et al. [1981], Houck and Datta [1981], Loucks and Dorfman [1975], and Stedinger et al. [1983].

In this paper a real-time operation model is proposed that uses chance constraints, assumes a linear decision rule (nonrestrictive) as an operation policy, and incorporates the probabilistic nature of real-time forecasts by considering the distribution of actual streamflow volumes conditioned on forecasted values. The coffititional distribution functions are constructed from the distribution of the errors associated with such forecasts. The objective of the model is the minimization of weighted probable deviations from storage and release targets obtained from a planning model. These weights are surrogates for the actual loss functions, and the probable deviations are functions of the reliability levels specified in the model.

The use of the long-term target values for release and storage makes the model capable of specifying a release policy that is a subset of a seasonal policy, thus eliminating the myopic (short-sighted) nature of operation. This model is also 
shown to be applicable to a system of reserviors. The restrictions associated with the use of a linear decision rule are shown to be invalid for this model.

\section{DEVELOPMENT OF THE MODEL}

Formulation of this model consists of two distinct steps. The first step involves the statistical evaluation of available forecasts for the inflows to the reservoir. The second step involves the formulation of the optimization model incorporating the statistical characteristics of the forecast errors.

\section{Incorporation of Hydrologic Forecast Errors}

The model to be developed requires streamflow forecasts and information on the reliability of these forecasts: The necessary statistical analysis includes the determination of the errors involved in forecasting the streamflow values for various time steps. These forecasts may be based on a stochastic model and/or a conceptual model. It is assumed that the errors have certain statistical properties that remain invariant for the time horizon for which the forecasts are made and for the time horizon of the optimization model.

One method of quantifying the reliability of forecasts begins with the estimation of the fractional error $e$ for a given time interval:

$$
e=\frac{\text { actual streamflow minus forecasted streamflow }}{\text { forecasted streamflow }}
$$

This definition implies that the errors in forecasts are normalized by the magnitude of respective forecasts. This definition may be modified, and the error considered may be assumed to be independent of the magnitude of forecasts for different cases, especially when a time series model is being used for forecasting and where the errors, as a requirement, are white noise.

From the existing streamflow records and the available forecasted streamflows for a portion of the record it is possible to construct the cumulative distribution function of $e$. This can be used to define the cumulative distribution function of the actual inflow, $R_{t}\left(f_{t}\right)$, during a period of $t$ days with a forecast of $f_{t}$ because

$$
R_{t}\left(f_{t}\right)=e f_{t}+f_{t}
$$

This distribution function is the basis of assessing the reliability that the actual streamflows will remain within given ranges. The following optimization model is then formulated by utilizing this information.

\section{The Optimization Model}

This optimization model utilizes the forecasted streamflow volumes and the distribution function of the actual streamflows conditioned on forecasted values. The explicit objective of the model is to meet as closely as possible the planning target values for release and storage for the time horizon of the model. The implicit objectives are to satisfy given lower and upper bounds of storage and release with specified reliabilities. The following notation is used in the model.

$b_{t}\left(f_{t}\right)$ decision variable for the period between the beginning of day 1 and the beginning of day $t+1$, conditioned on forecasted streamflow equal to $f_{\mathrm{t}}$ during the same period, $\mathrm{m}^{3}$;

$C A P$ capacity of reservoir, $\mathrm{m}^{3}$;

$D^{r}(t, \hat{t})$ release deficit from target value for the period between the beginning of day $t+1$ and the beginning of day $\hat{t}+1, \mathrm{~m}^{3}$;
$D_{i}^{s}$ storage deficit from target value at end of day $t$, $\mathrm{m}^{3}$;

$E^{r}(t, \hat{t})$ release excess from target value for the period between the beginning of day $t+1$ and the beginning of day $\hat{t}+1, \mathrm{~m}^{3}$

$E_{t}{ }^{s}$ storage excess from target value at end of day $t$, $\mathrm{m}^{3}$;

$f_{t}$ forecasted streamflow for the period between the beginning of day 1 and the beginning of day $t+1$, $\mathrm{m}^{3}$;

$F_{t}\left(\right.$ ) cumulative distribution function of $R_{d}\left(f_{t}\right)$;

$F_{t}^{-1}\left(\right.$ ) inverse cumulative distribution function of $R_{t}\left(f_{t}\right)$

$K(t, t)$ a tolerance limit placed on the deviation of release commitments obtained as solutions on a particular day for release in the period between the beginning of day $t+1$ and the beginning of day $\hat{t}+1$ from the release commitment for the same period obtained from the solution on the previous day, $\mathrm{m}^{3}$;

Pr probability of;

$P_{t}{ }^{\text {sd }}$ a constant equal to the weight specified in the objective function for the storage deficit from the target value at end of day $t$;

$p_{t}{ }^{\text {se }}$ a constant equal to the weight specified in the objective function for the storage excess from the target value at end of day $t$;

$p_{r i}{ }^{r d}$ a constant equal to the weight specified in the objective function for the release deficit from the target release in the period between the beginning of day $t+1$ and the beginning of day $\hat{t}+1$;

$p_{t t}{ }^{r e}$ a constant equal to the weight specified in the objective function for release excess from the target release in the period between the beginning of day $t+1$ and the beginning of day $\hat{t}+1$;

$R_{t}\left(f_{t}\right)$ streamflow between the beginning of day 1 and the beginning of day $t+1$, conditioned on forecasted streamflow equal to $f_{t}$ during the same time period, $\mathrm{m}^{3}$;

$S_{1} \quad$ initial storage at beginning of day $1, \mathrm{~m}^{3}$;

$S_{\min }$ minimum storage allowable, $\mathrm{m}^{3}$;

$S_{t+1}\left(f_{t}\right)$ storage at the beginning of day $t+1$, conditioned on forecasted inflow equal to $f_{t}$ in the time period from the beginning of day 1 to the beginning of day $t+1, \mathrm{~m}^{3}$;

$t$ th day;

$T$ time horizon considered, days;

$T A R$ target storage during the time horizon considered, $\mathrm{m}^{3}$;

$T_{R R}(t, t)$ target release between the beginning of day $t+1$ and the beginning of day $\hat{t}+1, \mathrm{~m}^{3}$;

$X_{\min }(t, \hat{t})$ minimum allowable release for the period between the beginning of day $t+1$ and the beginning of day $\hat{t}+1, \mathrm{~m}^{3}$

$X_{t}\left(f_{t}\right)$ release between the beginning of day 1 and the beginning of day $t+1$, conditioned on forecasted streamflow equal to $f_{t}$ in the same time period, $\mathrm{m}^{3}$;

$X(t, \hat{t})=X_{t}\left(f_{t}\right)-X_{t}\left(f_{t}\right)$ release between the beginning of day $t+1$ and the beginning of day $\hat{t}+1$, conditioned on forecasted streamflow equal to $f_{t}$ in the period between the beginning of day 1 and the beginning of day $t+1$ and equal to $f_{i}$ between the beginning of day 1 and the beginning of day $\hat{t}+1$ $(\hat{t} \geq t), \mathrm{m}^{3}$;

$\alpha(t, \hat{t}), \beta(t), \gamma(t), \delta(t), \mu(t)$ as defined by the constraints. 
The explicit form of the objective function considered in this model is the minimization of the weighted sum of maximum probable deviations from storage and release targets. These maximum probable deviations are defined by the reliabilities with which these deviations are not to be exceeded. A linear equivalent of this objective function suitable for inclusion in a linear program is

$$
\text { Minimize } \begin{aligned}
D & =\sum_{t=1}^{T}\left(P_{t}^{s d} * D_{t}^{s}+P_{t}^{s e} * E_{t}^{s}\right) \\
& +\sum_{t=0}^{T-1} \sum_{i=t+1}^{T}\left[P_{t i}^{r d} * D^{r}(t, \hat{t})+P_{t i}^{r e} * E^{r}(t, \hat{t})\right]
\end{aligned}
$$

The values of $D_{t}^{s}$ and $E_{t}{ }^{s}$ are deficit and excess storages at the end of day $t$, and the values of $D^{r}(t, \hat{t})$ and $E^{\prime}(t, \hat{t})$ are the deficit and excess releases for the period between the beginning of day $t+1$ and the beginning of day $\hat{t}+1$. The appropriate constraints to define the excess and deficit storage values are

$$
\begin{gathered}
S_{\mathrm{t}+1}\left(f_{\mathrm{t}}\right)= \\
D_{t}^{s} \geq 0 \\
E_{t}^{s} \geq 0
\end{gathered}
$$

Use of expected values in the objective function of a realtime operations model is restrictive in the sense that it requires utility functions that reflect how prone or averse the decision makers are to risk. Though theoretically plausible, in practice, utility functions are difficult if not impossible to construct because of the presence of multiple objectives, conflicting interests, and multiple decision makers. Therefore, the expected value criterion is not used in the objective function of this model.

The decision rule used assumes the release from the beginning of day 1 to the beginning of day $t+1$, conditioned on forecasted streamflow equal to $f_{t}$, to be a linear function of the storage at the beginning of day 1 :

$$
X_{t}\left(f_{t}\right)=S_{1}-b_{t}\left(f_{t}\right)
$$

The value of the decision variable $b_{t}\left(f_{t}\right)$ will be chosen to optimize the objective function.

Substituting the decision rule $X_{t}\left(f_{t}\right)=S_{1}-b_{t}\left(f_{t}\right)$ in the continuity or mass balance equation, the following equations are obtained:

$$
\begin{gathered}
S_{t+1}\left(f_{t}\right)=S_{1}+R_{t}\left(f_{t}\right)-X_{t}\left(f_{t}\right)=R_{t}\left(f_{t}\right)+b_{t}\left(f_{t}\right) \\
t=1,2, \ldots, T \\
X(t, \hat{t})=X_{i}\left(f_{t}\right)-X_{t}\left(f_{t}\right)=b_{t}\left(f_{t}\right)-b_{t}\left(f_{t}\right) \\
t=1,2, \ldots, T-1 \quad t<\hat{t} \leq T \\
X(0,1)=S_{1}-b_{1}\left(f_{1}\right)
\end{gathered}
$$

The chance constraints imposed, considering $R_{r}\left(f_{t}\right)$ as a random variable, are

1. The probability that the storage at the beginning of each period is greater than or equal to a specified value, $S_{\min }$, must equal or exceed a specified minimum value $\beta(t)$.

$$
\operatorname{Pr}\left[S_{t+1}\left(f_{t}\right) \geq S_{\min }\right] \geq \beta(t)
$$

or

$$
\operatorname{Pr}\left[R_{t}\left(f_{t}\right) \leq S_{\min }-b_{t}\left(f_{t}\right)\right] \leq 1-\beta(t)
$$

or

$$
F_{t}\left[S_{\min }-b_{t}\left(f_{t}\right)\right] \leq 1-\beta(t)
$$

or

$$
S_{\min }-b_{t}\left(f_{t}\right) \leq F_{t}^{-1}[1-\beta(t)] \quad t=1,2, \ldots, T
$$

2. The probability that reservoir storage at the beginning of each period will be less than the reservoir capacity $C A P$ must equal or exceed a specified value $\gamma(t)$. Apparently this equation means that there may be a nonzero probability of the reservoir capacity being exceeded. In reality this means that there will be a spill equal to the amount by which the capacity is exceeded.

$$
\operatorname{Pr}\left[S_{t+1}\left(f_{t}\right) \leq C A P\right] \geq \gamma(t)
$$

or

$$
C A P-b_{t}\left(f_{t}\right) \geq F_{t}^{-1}[\gamma(t)] \quad t=1,2, \ldots, T
$$

3. The probability that the release between the beginning of day $t+1$ and $\hat{t}+1$ is greater than the minimum specified value $X_{\min }(t, \hat{t})$ for that period must equal or exceed a specified minimum value $\alpha(t, \hat{t})$.

$$
\operatorname{Pr}\left[X_{t}\left(f_{t}\right)-X_{t}\left(f_{t}\right) \geq X_{\min }(t, \hat{t})\right] \geq \alpha(t, \hat{t})
$$

or

$$
\operatorname{Pr}\left[b_{t}\left(f_{t}\right)-b_{\hat{t}}\left(f_{k}\right) \geq X_{\text {min }}(t, \hat{t})\right] \geq \alpha(t, \hat{t})
$$

The quantity $X(t, \hat{t})$ is used rather than daily releases because the model assumes that the releases for shorter time periods are subsets of longer time period releases. The high degree of statistical dependence between inflows over these periods is accounted for in this way. Because $b_{t}\left(f_{t}\right)$ and $b_{t}\left(f_{k}\right)$ are not random variables for a particular solution, $\alpha(t, \hat{t})$ is assumed equal to 1 , so that the above constraints reduce to

$$
\begin{gathered}
b_{t}\left(f_{t}\right)-b_{t}\left(f_{t}\right) \geq X_{\min }(t, \hat{t}) \\
t=1,2, \ldots, T-1 \quad t<\hat{t} \leq T \\
S_{1}-b_{1}\left(f_{1}\right) \geq X_{\min }(0,1)
\end{gathered}
$$

Constraints 17 and 18 imply that the probabilities of meeting the release constraints are dependent on the probabilities of meeting the constraints on storages. These constraints ensure that $b_{t}\left(f_{t}\right) \geq b_{t}\left(f_{t}\right), t<\hat{t}$. If these constraints were not specified, it could have been possible that the releases specified by $X_{t}\left(f_{t}\right)$ would not be monotonically increasing functions of $t$. This is because different forecasts and error distributions are used for different values of $t(t=1, \ldots, T)$.

4. In order to account for the objective function given by equation (1), constraint sets that specify the maximum probable deviations from storage targets as a function of the specified reliability levels must be incorporated. Constraints serving this purpose may be specified as

$$
\operatorname{Pr}\left[S_{t+1}\left(f_{t}\right) \geq T A R-D_{t}^{s}\right] \geq \delta(t)
$$

or

$$
\operatorname{Pr}\left[R_{t}\left(f_{t}\right)+b_{t}\left(f_{t}\right) \geq T A R-D_{t}{ }^{\top}\right] \geq \delta(t)
$$

or

$$
\begin{array}{r}
T A R-D_{t}{ }^{s}-b_{t}\left(f_{t}\right) \leq F_{\mathrm{t}}{ }^{-1}[1-\delta(t)] \\
t=1,2, \ldots, T
\end{array}
$$


Similarly, for storage excesses from the target value,

$$
\operatorname{Pr}\left[S_{t+1}\left(f_{t}\right) \leq T A R+E^{s}(t)\right] \geq \mu(t)
$$

or

$$
\begin{array}{r}
T A R+E^{\mathrm{s}}(t)-b_{t}\left(f_{t}\right) \geq F_{\mathrm{t}}^{-1}[\mu(t)] \\
t=1,2, \ldots, T
\end{array}
$$

5. Because of this model's formulation, the release may be met with $100 \%$ probability, provided there is enough water in storage. Constraints that specify the deficit or excess releases to be incorporated in the objective function may be stated as

$$
X(t, \hat{t}) \geq T_{R R}(t, \hat{t})-D^{r}(t, \hat{t})
$$

or

$$
\begin{array}{r}
T_{R R}(t, t)-b_{t}\left(f_{t}\right)+b_{t}\left(f_{t}\right) \leq D^{\prime}(t, \hat{t}) \\
t=1,2, \ldots, T-1 \quad t<\hat{t} \leq T
\end{array}
$$

and

$$
T_{R R}(0,1)-S_{1}+b_{1}\left(f_{1}\right) \leq D^{r}(0,1)
$$

Also

$$
X(t, \hat{t}) \leq T_{R R}(t, \hat{t})+E^{\prime}(t, \hat{t})
$$

or

$$
\begin{aligned}
b_{t}\left(f_{t}\right)-b_{t}\left(f_{t}\right)- & T_{R R}(t, \hat{t}) \leq E^{r}(t, \hat{t}) \\
t & =1,2, \ldots, T-1 \quad t<\hat{t} \leq T
\end{aligned}
$$

and

$$
S_{1}-b_{1}\left(f_{1}\right)-T_{R R}(0,1) \leq E^{\prime}(0,1)
$$

6. The primary function of this model is predictive in nature; it may therefore be desirable that the proposed release policy for the next several days not change too dramatically as the release policy is updated during these several days. By limiting the changes in release policy from one day to the next, the flexibility to respond to streamflow forecast changes is reduced, but the ability to plan activities dependent on the release policy is enhanced. To restrict the deviations between the releases predicted for the same day or days by a previous solution of the model and a current solution of the model, these constraints may be added:

$$
\begin{aligned}
& X(t, \hat{t}) \leq X^{b}(t, \hat{t})+K(t, \hat{t}) \\
& X(t, \hat{t}) \geq X^{b}(t, \hat{t})-K(t, \hat{t})
\end{aligned}
$$

$X^{b}(t, \hat{t})$ denotes the optimum value of the predicted release between the beginning of day $t+1$ and the beginning of day $\hat{t}+1$ obtained as solution to the model on the previous day. In practice the value of $t$ may be restricted to 1 or 2 days, and the value of $\hat{t}$ may be restricted to $t+1$ or $t+2$ in these constraints. Real-time forecasts are quite accurate only for a lead time of 1 or 2 days, and restricting the operation policy to a function of the policy based on forecasts of longer intervals may not be advantageous. Theoretically, however, these constraints may be extended to cover the whole time horizon incorporated in a particular solution. Equivalent constraints using the linear decision rule may also be stated as:

$$
\begin{array}{r}
b_{\mathrm{t}}\left(f_{t}\right)-b_{\mathrm{t}}\left(f_{\mathrm{t}}\right) \leq X^{b}(t, \hat{t})+K(t, \hat{t}) \\
t=1,2, \ldots, T-1 \\
S_{1}-b_{1}\left(f_{1}\right) \leq X^{b}(0,1)+K(0,1)
\end{array}
$$

and

$$
\begin{aligned}
b_{t}\left(f_{t}\right)-b_{t}\left(f_{t}\right) & \geq X^{b}(t, \hat{t})-K(t, \hat{t}) \\
t & =1,2, \ldots, T-1 \quad t<\hat{t} \leq T \\
S_{1}-b_{1}\left(f_{1}\right) & \geq X^{b}(0,1)-K(0,1)
\end{aligned}
$$

Therefore, this model consists of the objective function (equation (1)) of minimizing the weighted sum of maximum probable deviations from target values of releases and storages for the time horizon of the model and a set of constraints (12, $14,17,18,21,23,25,26,28,29,32-35$ ) on performance requirements and the reliabilities with which these performance criteria are to be met. The forecasts required for a single day's solution of this optimization model are one time-step ahead forecasts for time periods varying from 1 day to the time horizon, which may be about 30 days. For each time step, different forecasting models may be used.

The objective function can be generalized to represent better the actual penalty (opportunity cost) for deviations from the target values if $P_{t}^{s d}, P_{t}^{s e}, P_{t t}{ }^{r d}$, and $P_{t t}{ }^{r e}$ are defined as functions instead of constants. The discounting factors for uncertainties in the future may be assumed to be included in these functions. Using these functions instead of the weights (constants) as described before will require their definition in the constraint set and most likely require piecewise linearization because they will be nonlinear.

A further generalization of the objective is possible by allowing the reliability levels $(\beta, \alpha, \mu)$ to be variables. Then the penalty functions would not only be functions of storage and releases but also the reliabilities of those storages and releases. Appropriate ranges of the cumulative distribution functions (CDF) of actual inflows conditioned on forecasted flows would have to be included (piecewise linearized) in the constraint set. One last extension of the objective would be to include the entire CDF's of storages and releases in the objective. This could be done approximately by defining several reliability levels $(\beta, \alpha, \mu)$ between 0 and 1 , determining the associated storage or release values, and approximating the remainder of the CDF's.

In the next section the solution of the model will be discussed. The results of simulating the operation policies in a simulation model and motivations for some of the results will be presented. It is hoped that this evaluation will help to make the assumptions in the model more clear.

\section{SOlution OF THE MOdel}

The first step in the evaluation of this model was to establish a hypothetical reservoir system for test purposes. In practice the capacity of the reservoir will be a known value; however, because the reservoir system used in this evaluation is hypothetical, a single linear decision rule (LDR) model [ReVelle et al., 1969] with 12 seasons per year was used to find the optimal capacity reservoir for the tests. The capacity required for this hypothetical reservoir on the Tygert River in West Virginia was $168.7 * 10^{6} \mathrm{~m}^{3}$.

The next step in the model construction was the development of the forecasting models for different time steps Simple autoregressive models were used because the primary purpose of the optimization model presented here is not to evaluate the degree of accuracy of the forecasting models but to utilize the information on the probable errors associated with the forecasts in evaluating the reliability of operations. To preserve the seasonal characteristics of the streamflow se- 
TABLE 1. Weights for the Objective Function

\begin{tabular}{|c|c|}
\hline $\begin{array}{c}\text { Weights on } \\
\text { Storage Deviations }\end{array}$ & $\begin{array}{l}\text { Weights on } \\
\text { Release Deviations }\end{array}$ \\
\hline $\begin{aligned} P_{1}{ }^{s d} & =0.31 \\
P_{2}^{s d} & =0.32 \\
P_{3}^{s d} & =0.33 \\
P_{7}^{s d} & =0.40 \\
P_{30}{ }^{s d} & =0.50 \\
P_{1}{ }^{s e} & =0.30 \\
P_{2}^{s e} & =0.30 \\
P_{3}{ }^{s e} & =0.30 \\
P_{7}{ }^{s e} & =0.40 \\
P_{30}{ }^{s e} & =0.50\end{aligned}$ & $\begin{aligned} P_{01}{ }^{r d} & =1.2 \\
P_{12}{ }^{r d} & =0.9 \\
P_{23}^{r d} & =0.6 \\
P_{37}{ }^{r d} & =0.3 \\
P_{730}{ }^{r d} & =0.1 \\
P_{01}^{r e} & =1.2 \\
P_{12}^{r e} & =0.9 \\
P_{23}^{r e} & =0.6 \\
P_{37}^{r e} & =0.3 \\
P_{730}{ }^{r e} & =0.1\end{aligned}$ \\
\hline
\end{tabular}

quences, different forecasting models may be developed for different periods of the year.

The use of a linear decision rule in the formulation of this model does not limit the solution space as it does in other models. $S_{1}$ is always a known quantity in an operation model, and therefore $b_{t}\left(f_{t}\right)$ can easily be replaced by $S_{1}-X_{t}\left(f_{t}\right)$. Equation (12) would become

$$
S_{\min }-S_{1}+X_{t}\left(f_{t}\right) \leq F_{t}^{-1}[1-\beta(t)]
$$

It is now possible to find the optimum value of the decision variables $X_{t}\left(f_{t}\right)$ and $X(t, \hat{t})$ by utilizing (17). This would not be the case with a planning model where $S_{1}$ is the initial storage at the beginning of a season and is to be treated as a random variable. The limitiations often cited when a linear decision rule (LDR) is used should not be valid here. This model should serve as an example where use of an LDR is not at all restrictive.

The optimization model already presented is a generalized version of the model that was solved. To limit the computational burden and to approximate more accurately the actual decision-making environment, the number of CDF's to be considered in a time horizon of $T$ days was limited. For this particular study, according to the notation used, the values of $t$ were restricted to $0,1,2,3,7$, and 30 days. Values of $\hat{t}$ were restricted to $1,2,3,7$, and 30 days only. Also for constraints 32 and 34 the value of $t$ was restricted to 0,1 , and 2 days, while the value of $\hat{t}$ was restricted to 1,2 , and 3 days. This means that in each subsequent solution of this model, at the beginning of each day, the release policy for the first three days of solution was restricted to within some specified tolerance limits of the predicted release as obtained by a solution of this model for the previous day.

This model also requires as inputs the storage volume at the beginning of each day and the updated forecasts made on a real-time basis at the beginning of each day. The releases and storages for the second, third, and later days as specified by the model are only used for forecasting purposes to predict the releases in future time periods. Only the solution for the first day is used for making actual releases. This preserves the realtime characteristics of the operation policy. The release policy as predicted for a longer time period may be restricted by introducing bounds into the model to maintain the releases for a given time horizon or for a particular season within some limits of the release commitment made from a planning model.

The objective weights used in this example $\left(P_{t}{ }^{\text {sd }}, P_{t}{ }_{t}^{\text {se }}, P_{t}{ }^{\text {rd }}\right.$, and $P_{t i}{ }^{r e}$ ) were chosen arbitrarily (Table 1 ), keeping in mind the general assumption that deviations from release targets are more costly than an equal amount of deviation from a storage target. Also the meeting of targets on the first day of a particular solution is relatively more important than that of subsequent days when the model inputs are actually updated and the operation policy revised.

The model was solved for varying initial conditions and different reliability levels. The operation policies obtained as solutions were tested by simulation of reservoir operation. The mathematical programming package XMP [Marsten, 1980] was used to solve the linear optimization models. Some of the many variations of the model that were tested are given in Table 2. These models were tested for an identical set of streamflow data for a period of $\mathbf{3 0}$ days. The daily flows actually occurring consisted of a series of high flows beginning on the third day and ending on the seventh day and a series of low flows beginning on the twenty-second day. Tables 2 and 3 summarize some of the results obtained.

Table 2 shows some of the variations of the model that were tested. The columns in Table 2 specify the various levels of reliabilities that were used in these models. In model $\mathbf{A}$, only the predicted values of streamflow were used. Therefore no reliability levels are applicable to this model. Model C specifies lower reliability levels as compared to model B for all the performance requirements except the storage deviation from target value in the 7-and 30-day periods.

Table 3 shows some results of solving these models at the beginning of each day for a 30-day period and of simulating operating conditions by using actual streamflow volumes. Because a series of high flows was considered for these examples, the maximum volumes of the releases and storages were considered critical. It is evident from Table 3 that the maximum storage attained during this period is largest in model $\mathrm{A}$. While model $C$ results in a smaller value of excess deviation from release target compared to model $B$, this occurs at the expense of attaining a higher storage excess than model $\mathbf{B}$. This is because in model $\mathbf{B}$ the deviations from storage target are restricted with higher reliabilities; however, in the process of complying with this requirement, model $B$ is forced to release a larger amount in order to be risk averse, i.e., to avoid

TABLE 2. Identification of Models With Different Inputs

\begin{tabular}{ccccccccccccc}
\hline $\begin{array}{c}S_{1} \text { on } \\
\text { Model } \\
\text { Identity }\end{array}$ & $\begin{array}{c}\text { First Day, } \\
10^{6} \mathrm{~m}^{3}\end{array}$ & $\begin{array}{c}X_{\min }(0,1) \\
10^{6} \mathrm{~m}^{3}\end{array}$ & $\beta_{1}, \gamma_{1}$ & $\beta_{2}, \gamma_{2}$ & $\beta_{3}, \gamma_{3}$ & $\beta_{7}, \gamma_{7}$ & $\beta_{30}, \gamma_{30}$ & $\delta_{1}, \mu_{1}$ & $\delta_{2}, \mu_{2}$ & $\delta_{3}, \mu_{3}$ & $\delta_{7}, \mu_{7}$ & $\delta_{30}, \mu_{30}$ \\
\hline A & 85.57 & 2.44 & $-\bar{N}^{*}$ & - & - & - & - & - & - & - & - & - \\
B & 85.57 & 2.44 & 0.90 & 0.85 & 0.85 & 0.75 & 0.70 & 0.85 & 0.85 & 0.80 & 0.70 & 0.70 \\
C & 85.57 & 2.44 & 0.75 & 0.75 & 0.75 & 0.70 & 0.70 & 0.75 & 0.75 & 0.75 & 0.75 & 0.75 \\
\hline
\end{tabular}

$C A P=168.70^{*} 10^{6} \mathrm{~m}^{3} ; T_{R R}(t, \hat{t})=(\hat{t}-t)^{*} 2^{*} X_{\min }(0,1) ; S_{\min }=24.45^{*} 10^{6} \mathrm{~m}^{3} ; T A R=73.35^{*} 10^{6} \mathrm{~m}^{3} ;$

$K(t, \hat{t})=T_{R R}(t, \hat{t}) ; X_{\min }(t, \hat{t})=(\hat{t}-t)^{*} X_{\min }(0,1)$.

${ }^{*}$ Used predicted values only. $\beta, \gamma, \delta, \mu$ values not applicable. 
TABLE 3. Simulation and Optimization Results for Different Models

\begin{tabular}{ccccccc}
\hline Model & $\begin{array}{c}\text { Maximum } \\
\text { Storage, } \\
\text { Identity }\end{array}$ & $\begin{array}{c}\text { Maximum } \\
\text { Daily } \\
\text { Release, } \\
10^{6} \mathrm{~m}^{3}\end{array}$ & $\begin{array}{c}\text { Ending } \\
\text { Storage } \\
\text { on 10th } \\
\text { Day, } \\
10^{6} \mathrm{~m}^{3}\end{array}$ & $\begin{array}{c}\text { Percentage of } \\
\text { Time Maximum } \\
\text { Predicted Storage } \\
\text { Deviation Exceeded } \\
\text { in Initial 10 Days }\end{array}$ & $\begin{array}{c}\text { Maximum } \\
\text { Inflow } \\
\text { on 5th } \\
\text { Day, } \\
10^{6} \mathrm{~m}^{3}\end{array}$ & $\begin{array}{c}\text { Ending } \\
\text { Storage } \\
\text { on 30th } \\
\text { Day, } \\
10^{6} \mathrm{~m}^{3}\end{array}$ \\
\hline A & 133.64 & 27.62 & 77.11 & 30.0 & 26.65 & 55.55 \\
B & 123.52 & 20.41 & 91.78 & 20.0 & 26.65 & 68.01 \\
C & 130.67 & 16.33 & 93.74 & 30.0 & 26.65 & 71.02 \\
\hline
\end{tabular}

bigger deviations in the future. Also the ending storages after 30 days (which is also the time horizon of the models for a particular solution) are nearer to the target value for models $B$ and $\mathrm{C}$ than for model $\mathrm{A}$.

The inadequacy of using only predicted values as deterministic inputs to the model is evident from the performance of model A. Because this model simply uses the predicted values, whenever high flows occur that differ appreciably from the predicted value, this model fails to recognize the higher percentile values of future probable flows. This may result in very high deviations from the target values at certain stages. Also, for a series of low flows, when not enough water is available to meet the release targets during the last few days, the storage is depleted faster than with the other two models.

Figures 1-3 show release patterns obtained for these three different models for an identical set of inflows. The fluctuations in release volumes and storage volumes are most pronounced in model $\mathrm{A}$. Model $\mathrm{C}$ shows the minimum fluctuation. With uncertain inputs, very large probable deviations from target values are guarded against in models $B$ and $C$ with higher reliabilities than model $\mathrm{A}$. However, in model $\mathrm{C}$ the reliability levels are smaller than those of model $B$, and therefore the critical values of minimum or maximum inflows considered probable are less critical in model $\mathrm{C}$ than in model B. This may cause model $B$ to release a higher volume than model $\mathbf{C}$ when a high flow is forecasted with identical initial conditions.

Although many other variatons of the model with different inputs were considered, they are not reported here because it is not possible to reach definitive conclusions from limited examples. It is especially true when the performance of the model is very much dependent on the quality of the forecasts obtained from the forecasting model. This aspect is discussed in greater detail in Datta and Burges [1984]. These evaluations only show that the model is solvable, it gives sensible results that are not counterintuitive, and these evaluations may be used to aid the judgment of the decision makers.

\section{Extension of the Model to a Series of Reservoirs}

One of the strong points of the model presented is that it does not require complicated statistical manipulations such as convolution. Also, the linear programming algorithm can be used. This algorithm is simpler to use and substantially more versatile in many situations than other solution approaches such as dynamic programming. Therefore this model should be very useful if it can be extended to a series or network of reservoirs and still remain well within the range of computational feasibility. The modification necessary to use this model for a system of reservoirs in series is presented here as an illustration. The objective function is now modified to

$$
\text { Minimize } \begin{aligned}
D= & \sum_{t=1}^{T} \sum_{i=1}^{N}\left(P_{i t}^{s d} * D_{i t}^{s}+P_{i t}^{s e} * E_{i t}^{s}\right) \\
& +\sum_{t=0}^{T-1} \sum_{t=1+1}^{T} \sum_{i=1}^{N} \\
& \cdot\left[P_{i t t}^{r d} * D_{i}^{r}(t, \hat{t})+P_{i t t}^{r e} * E^{r}(t, \hat{t})\right]
\end{aligned}
$$

Here the subscript $i$ denotes reservoir $i$, with $N$ reservoirs in series; for this example the reservoirs are numbered starting with 1 as the most upstream reservoir down to $N$ as the most downstream reservoir. All the weights $P$ may again be replaced by actual or hypothetical loss or penalty functions for deviations from storage and release targets.

The continuity or mass balance equation is to be modified to reflect that the total inflow into a reservoir is the uncontrolled inflow from the basin plus the release from an upstream reservoir minus any withdrawal by the users between reservoirs. Therefore $X(t, \hat{t})$ should now be replaced by $X_{i}(t, \hat{t})$ and $Y_{i}(t, \hat{t}): X_{i}(t, \hat{t})$ refers to that part of the release (with-

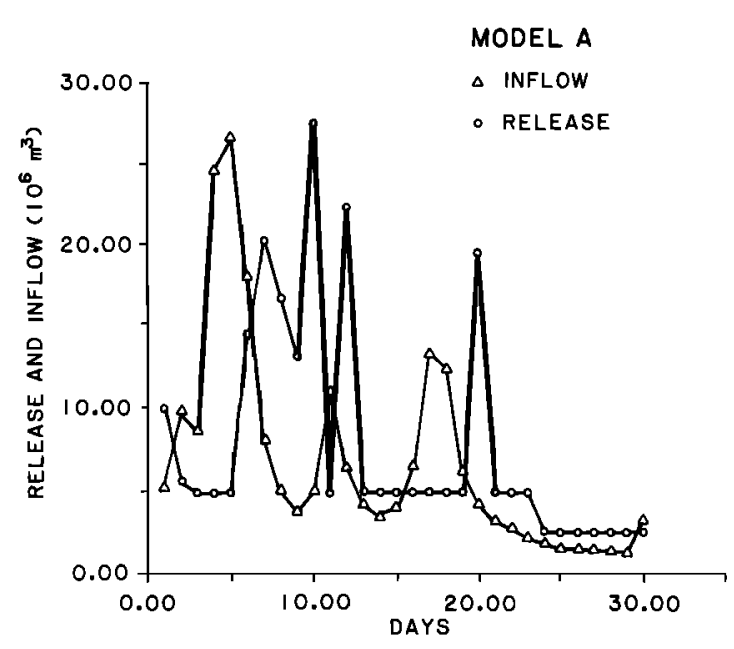

Fig. 1. Releases and inflows for model A.

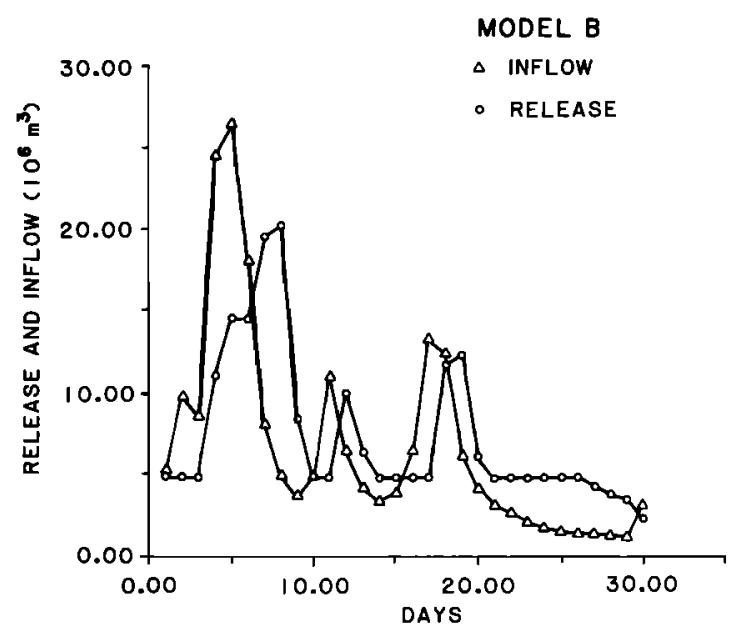

Fig. 2. Releases and inflows for model B. 


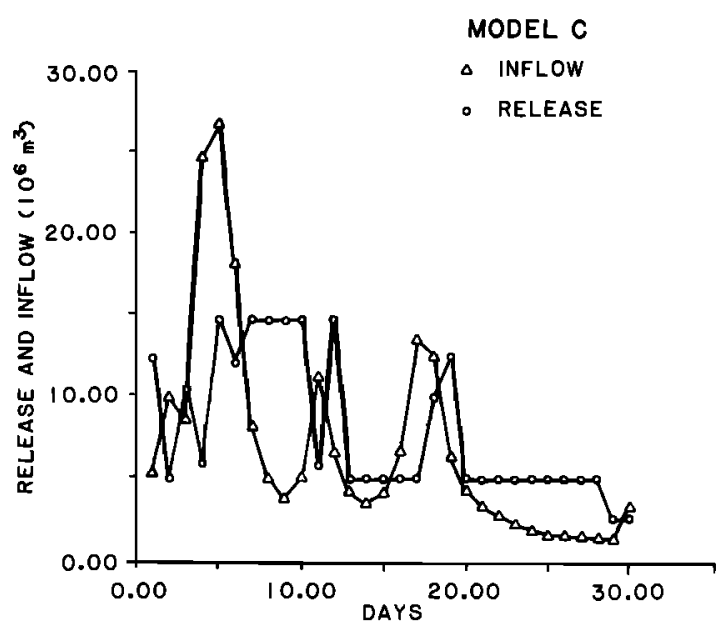

Fig. 3. Releases and inflows for model C.

drawal) from reservoir $i$ used in the control area of reservoir $i$; and $Y_{i}(t, \hat{t})$ refers to that portion of the release from reservoir $i$ that enters the downstream reservoir $i+1$ as inflow. If the losses incurred as a result of deviations from a release target are due solely to the withdrawal from a reservoir $X_{i}(t, \hat{t})$, then the deviations from the target withdrawal for a particular reservoir will be determined by $T_{R R_{I}}(t, t)-X_{i}(t, \hat{t})$.

For purposes of this illustration the release from a reservoir is assumed to arrive at the next downstream reservoir in the same day. Therefore, equations (5)-(7) are now given as:

$$
\begin{aligned}
& X_{i t}\left(f_{t}\right)+Y_{i t}\left(f_{i t}\right)=S_{i 1}-b_{i t}\left(f_{t t}\right) \\
& S_{i, t+1}\left(f_{i t}\right)= S_{i 1}+R_{i t}\left(f_{i t}\right)-X_{i t}\left(f_{i t}\right)-Y_{t t}\left(f_{i t}\right) \\
&+Y_{2-1, t}\left(f_{i-1, t}\right) \quad t=1,2, \ldots, T \quad i=2, \ldots, N
\end{aligned}
$$

For $i=1$ the previous equations become

$$
S_{1, t+1}\left(f_{1 t}\right)=S_{1 t}+R_{1 \mathrm{t}}\left(f_{1 \mathrm{t}}\right)-X_{1 \mathrm{t}}\left(f_{1 \mathrm{t}}\right)-Y_{1 \mathrm{t}}\left(f_{1 \mathrm{t}}\right)
$$

and

$$
\begin{gathered}
X_{i}(t, \hat{t})+Y_{i}(t, \hat{t})=b_{i t}\left(f_{i t}\right)-b_{i t}\left(f_{i z}\right) \\
t=1,2, \ldots, T-1 \quad t<\hat{t} \leq T \quad i=1, \ldots, N
\end{gathered}
$$

Other constraints are to be suitably modified. For example, constraint 12 will now be given by

$$
\begin{aligned}
S_{\text {min }}-b_{i t}\left(f_{i t}\right)-Y_{i-1, t}\left(f_{i-1, t}\right) & \leq F_{i t}{ }^{-1}[1-\beta(t)] \\
t & =1,2, \ldots, T \quad i=2, \ldots, N
\end{aligned}
$$

Constraint 25 will now be given by

$$
\begin{gathered}
T_{R R_{i}}(t, \hat{t})-b_{i t}\left(f_{i z}\right)+b_{i t}\left(f_{i t}\right)+Y_{i}(t, \hat{t}) \leq D_{i}{ }^{\prime}(t, \hat{t}) \\
t=1,2, \ldots, T-1 \quad t<\hat{t} \leq T \quad i=1, \ldots, N
\end{gathered}
$$

where $Y_{i}(t, \hat{t})$ is defined by

$$
Y_{i}(t, \hat{t})=Y_{i z}\left(f_{i z}\right)-Y_{i t}\left(f_{i t}\right)
$$

In a similar fashion, all other constraints can be modified. When there are reservoirs in series and parallel, similar constraints can be formulated.

\section{Summary and Conclusions}

This model is primarily for use as a real-time daily operation model. It must be solved at the beginning of each day, with updated forecasts, revised conditional distribution functions of future streamflows, and the state of the system given by the initial storage used as inputs. The objective of operation is to minimize the sum of penalties associated with deviations from target or ideal conditions for operations over a time horizon of several days to a month.

Theoretically, this model can be extended to shorter timesteps such as hourly operations. However, hourly operations are generally based on almost perfect forecasts of streamflows and exact consumer demands for water supply and hydroelectric power. Therefore in such a situation a deterministic optimization model, rather than a chance-constrained model, may be more appropriate. Operation policies obtained as solutions from monthly or seasonal models may be used as planning inputs to the daily operation model so that the release in a time horizon of 30 days can be specified by appropriately fixing the value of the decision parameter $b_{T}\left(f_{T}\right)$ with some tolerance.

The solutions obtained from the optimization model for different input conditions were studied in a simulation of reservoir operation by using these policies. The results obtained give some insight to the working of this model. The simulation results may aid in the selection of appropriate levels of the reliabilities to be specified by the decision makers for meeting different operational requirements. Also, using only the forecasted values and ignoring the uncertain parts is a special case of this model and may be acceptable when the probabilities of system failures are very low. Some limitations of using only a forecasted value were demonstrated through model $\mathrm{A}$.

The time horizon that should be considered in a particular solution of this model must be decided on the basis of the operational objectives. If the smaller time-step operation policies are intended to be subsets of longer time-step operation policies like seasonal or weekly time steps, the value of $T$ should be appropriately specified.

This model was shown to be extendable to a system of reservoirs. Also, the restrictions generally associated with the use of linear decision rules were shown to be invalid for this model.

Acknowledgments. This material is based upon work supported by The National Science Foundation under grant CME-7916819. The authors are also grateful to the Purdue Research Foundation for their generous financial support under grant XR-0340.

\section{REFERENCES}

Becker, L., and W. W-G. Yeh, Optimization of real time operation of multiple reservoir system, Water Resour. Res., 10(6), 1107-1112, 1974.

Becker, L., W. W-G. Yeh, D. Fults, and D. Sparks, Operation models for the Central Valley Project, J. Water Resour. Plann. Manage. Div., Am. Soc. Civil Eng., 102(WR1), 101-115, 1976.

$\mathrm{Chu}, \mathrm{W}$. S., and W. W-G. Yeh, A nonlinear programming algorithm for real time hourly reservoir operations, Water Resour. Bull., 14(5), 1048-1063, 1978.

Datta, B., and S. J. Burges, Short-term single, multipurpose reservoir operation: Importance of loss functions and forecast errors, Water Resour. Res., in press, 1984.

Fults, D. M., and L. F. Hancock, Optimum operations models for Shasta-Trinity system, J. Hydraul. Div., Am. Soc. Civil Eng., 98(HY9), 1497-1514, 1972.

Hogan, A. J., J. G. Morris, and H. E. Thomson, Decision problems under risk and chance constrained programming: Dilemmas in the transition, Manage. Sci., 27(6), 698-716, 1981. 
Houck, M. H., What is the best objective function for real-time optimal reservoir operation?, paper presented at International Symposium on Real Time Operation of Hydrosystems, Univ. Waterloo, Ontario, Canada, 1981.

Houck, M. H., and B. Datta, Performance evaluation of a stochastic optimization model for reservoir design and management, Water Resour. Res., 17(9), 827-832, 1981.

Jamieson, D. G., and J. C. Wilkinson, River Dee research program, 3 , A short-term control strategy for multipurpose reservoir systems, Water Resour. Res., 8(4), 911-920, 1972.

Joeres, E. F., J. S. Gunther, and H. M. Englemann, The Linear Decision Rule (LDR) reservoir problem with correlated inflows, 1, Model development, Water Resour. Res., 17(1), 18-24, 1981.

Loucks, D. P., and P. J. Dorfman, An evaluation of some linear decision rules in chance-constrained models for reservoir planning and operation, Water Resour. Res., 11(6), 777-782, 1975.

Marsten, R. E., The Design of the XMP Linear Programming Library, MIS Tech. Rep. 80-2, Univ. Ariz., Tucson, 1980.

ReVelle, C. S., E. Joeres, and W. Kirby, The linear decision rule in reservoir management and design, 1, Development of the stochastic model, Water Resour. Res., 5(4), 767-777, 1969.

Shane, M. R., and K. C. Gilbert, Weekly Time Step Reservoir System Scheduling Model, Part 1 and 2, report, Tenn. Val. Auth., Water Syst. Dev. Branch, Norris, Tenn., 1980.

Sigvaldason, $\mathrm{O}$. T., A simulation model for operating a multipurpose multireservoir system, Water Resour. Res., 12(2), 263-278, 1976.

Stedinger, J. R., B. F. Sule, and D. Pei, Multiple reservoir system screening models, Water Resour. Res., 19(6), 1383-1393, 1983.

Tennessee Valley Authority, Development of a comprehensive Tennessee Valley Authority water resource management program, paper presented at conference of International Institute for Applied Systems Analysis, Laxenberg, Austria, July 1974.

Toebes, G. H., and C. Rukvichai, Reservoir systems operating policy-Case study, J. Water Resour. Plann. Manage. Div., Am. Soc. Civil Eng., 104(1), 195-209, 1978.

Trott, W. J., and W. W-G. Yeh, Optimization of multiple reservoir systems, J. Hydraul. Div., Am. Soc. Civil Eng., 99(HY10), 1865-1884, 1973.

Windsor, J. S., Optimization models for the operating of flood control systems, Water Resour. Res., 9(5), 1219-1226, 1973.

Yazicigil, H., M. H. Houck, and G. H. Toebes, Daily operation of a multipurpose reservoir system, Water Resour. Res., 19(1), 1-14, 1983.

Yeh, W. W-G., State of the art review: Theories and applications of systems analysis techniques to the optimal management and operation of a reservoir system, Rep. UCLA-ENG-82-52, Univ. Calif., Log Angeles, 1982.

Yeh, W. W-G., L. Becker, and W. S. Chu, Real time hourly reservoir operations, J. Water Resour. Plann. Manage. Div., Am. Soc. Civil Eng., 105(WR2), 187-203, 1979.

B. Datta, Water Resources Management Laboratory, Engineering Experiment Station, University of Arkansas, Fayetteville, AR 72701.

M. H. Houck, School of Civil Engineering, Purdue University, West Lafayette, IN 47907.

(Received September 22, 1982; revised April 16, 1984; accepted May 7, 1984.) 